\title{
AVALIAÇÃO DE IMPACTOS E ADAPTAÇÃO ÀS MUDANÇAS CLIMÁTICAS: MODELOS DE ANÁLISE DO SETOR AGRÍCOLA ${ }^{1}$
}

\author{
Dênis Antônio da Cunha
}

Resumo: A maior parte dos estudos socioeconômicos que tratam das mudanças climáticas procura explicar qual é o custo associado a esse fenômeno, considerando os diferentes cenários propostos para o futuro. Tratando especificamente do setor agrícola, a literatura é diversificada e apresenta resultados distintos. Essas diferenças se devem, principalmente, aos métodos alternativos de análise, às condições climáticas tomadas como base, às previsões de crescimento econômico e aos cenários climáticos considerados. Neste artigo, procurou-se organizar essa literatura em duas abordagens principais. Uma delas procura entender o impacto sobre o setor agrícola, desconsiderando, ou apenas incluindo, implicitamente, as estratégias adaptativas; a segunda abordagem modela explicitamente a adaptação, analisando seu potencial de mitigar os efeitos negativos das mudanças climáticas. Concluiu-se que, mesmo havendo diferenças de entendimento sobre a forma de mensurar os impactos, a maior parte dos estudos prevê que as nações em desenvolvimento serão as mais afetadas negativamente pelos efeitos das mudanças climáticas globais.

Palavras-Chave: Mudanças Climáticas; Setor Agrícola; Adaptação; Metodologias Alternativas.

1 Recebido em: 21/01/13; Aceito em: 18/02/2013.

2 Doutor em Economia Aplicada. Professor Adjunto do Departamento de Economia Rural da Universidade Federal de Viçosa. E-mail: denis.cunha@ufv.br 


\begin{abstract}
Most socioeconomic studies that address climate change aims to explain what is the cost associated with this phenomenon, considering the different scenarios proposed for the future. Taking account specifically the agricultural sector, the literature is quite diverse and presents different results. These differences are mainly due to alternative methods of analysis, climatic conditions, the economic growth outlooks, and climate scenarios used. In this paper I tried to summarize this literature into two major approaches. The first one aims to understand the impact on the agricultural sector, disregarding, or only implicitly including, the adaptive strategies; the second approach is able to model explicitly the adaptation, analyzing their potential to mitigate the negative effects of climate change. It was concluded that, even with differences of understanding on how to measure the impacts, most studies predicts that developing countries will be the most negatively affected by global climate change effects.
\end{abstract}

Keywords: Climate Change; Agricultural Sector; Adaptation; Alternative Methodologies

\title{
1. Introdução
}

O termo mudança climática, conforme definido pela Convenção das Nações Unidas sobre Mudança do Clima (United Nations Framework Convention on Climate Change-UNFCCC, 2009), refere-se a qualquer alteração no clima consequente, direta ou indiretamente, da atividade humana, modificando a composição da atmosfera global, e que seja adicional à variabilidade climática natural observada em períodos de tempo comparáveis. Por sua vez, o IPCC (2007) define mudança climática como qualquer mudança no clima ocorrida ao longo do tempo, devido à variabilidade natural ou decorrente da atividade humana.

Independentemente de qual definição seja considerada, pode-se afirmar que a mudança climática é um fenômeno permanente, distinto das alterações que ocorrem nas condições do tempo no dia a dia. Segundo Fisher et al. (2009), tempo é o que ocorre num momento particular, sendo essencialmente de curto prazo, como temperatura e precipitação (realizações diárias, mensais, anuais etc.). Clima, por sua vez, é o padrão de longo prazo do tempo. Para cientistas climáticos, portanto, mudança climática é um fenômeno de longo prazo. 
O grau em que um sistema econômico é suscetível aos efeitos adversos da mudança climática, incluindo a variabilidade do clima e os eventos extremos, é chamado de vulnerabilidade. O nível de vulnerabilidade das diferentes regiões, setores econômicos e grupos sociais é determinado por fatores ambientais e socioeconômicos. Os fatores socioeconômicos incluem o nível de desenvolvimento tecnológico, a infraestrutura, as instituições e a organização política, enquanto os ambientais estão relacionados às características, à magnitude e à taxa de variação climática, ao qual o sistema está exposto, e à disponibilidade de recursos naturais. A capacidade adaptativa das regiões, também determinada por esses dois conjuntos de fatores, igualmente interfere no grau de exposição às mudanças climáticas (IPCC, 2007).

O setor agrícola, por depender diretamente de temperatura e precipitação, pode ser um dos mais afetados pelas mudanças climáticas, uma vez que, mesmo com todos os avanços tecnológicos, as condições ambientais ainda são fatores chave para a produtividade agrícola (DESCHÊNES; GREENSTONE, 2007; FISHER et al., 2009). O clima influencia tanto o crescimento e o desenvolvimento das plantas quanto as diversas etapas das atividades agrícolas, como o preparo da terra para semeadura, época de plantio, colheita, transporte e armazenamento dos produtos. As condições climáticas igualmente afetam a relação das plantas com doenças e pragas que prejudicam as culturas, causando perdas sociais e econômicas. Fenômenos meteorológicos adversos, de difícil previsibilidade em médio e longo prazo, como geadas, secas, chuva excessiva ou granizo, aumentam o risco associado à agricultura (GOUVÊA, 2008) ${ }^{3}$.

De acordo com Hanemann (2008), a maior parte dos estudos socioeconômicos que tratam das mudanças climáticas procura explicar

3 Deve-se notar que as atividades agropecuárias não apenas sofrem os efeitos das mudanças climáticas, mas também as influenciam. Segundo o IPCC (2007), os aumentos da concentração de metano e de óxido nitroso são devidos, principalmente, à agropecuária. Além disso, o setor é responsável por, aproximadamente, $13,5 \%$ das emissões anuais de $\mathrm{CO}_{2-e q}$ (gás carbônico equivalente, unidade-padrão que mede a capacidade de contribuir para o aquecimento global de todos os GEE, em quantidade de $\mathrm{CO}_{2}$ ). 
o custo associado a esse fenômeno, considerando os diferentes cenários propostos para o futuro. Tratando especificamente do setor agrícola, a literatura é vasta, diversificada e, em geral, apresenta resultados muito distintos. Essas diferenças se devem, principalmente, aos métodos alternativos de análise, às condições climáticas tomadas como base, às previsões de crescimento econômico e aos cenários climáticos considerados. Alguns estudos incluem adaptação, fertilização por carbono ${ }^{4}$ e/ou adoção de mudanças técnicas, ao passo que outros ignoram todos esses fatores; alguns consideram cenários climáticos antigos, muito pessimistas ou otimistas demais e são calibrados para espaços territoriais pequenos e bem definidos ou para vastos territórios (MENDELSOHN, 2000). Por essa razão, qualquer tentativa de síntese desses estudos seria incompleta.

Não obstante, é possível organizar essa literatura em duas abordagens principais. Uma delas procura entender o impacto sobre o setor agrícola, desconsiderando, ou apenas incluindo, implicitamente, as estratégias adaptativas, ao passo que a outra modela explicitamente a adaptação, analisando seu potencial de mitigar os efeitos negativos das mudanças climáticas. Portanto, no presente artigo, serão apresentados e discutidos os aspectos principais dessas diferentes metodologias.

Além dessa introdução, o artigo está dividido em outras três seções. Na segunda, serão descritos os modelos tradicionais de análise do impacto das mudanças climáticas sobre o setor agrícola; na terceira, serão abordados os modelos que consideram adaptação; por fim, na última seção, são feitas as considerações finais.

4 Evidências sugerem que muitas espécies vegetais cultivadas poderão responder positivamente ao aumento da concentração de $\mathrm{CO}_{2}$ na atmosfera, na ausência de outras condições de estresse (LONG et al., 2004). Entretanto, os efeitos benéficos da elevação da concentração de $\mathrm{CO}_{2}$ na atmosfera podem ser compensados por outros efeitos das mudanças climáticas, tais como o aumento da temperatura e alterações nos padrões de precipitação (EASTERLING et al., 2007). 


\section{Modelos tradicionais}

A metodologia pioneira nas análises de impactos das mudanças climáticas sobre o setor agrícola é conhecida como modelo agroeconômico ou abordagem da função de produção. O modelo é baseado em experimentos controlados que estimam a produtividade de cada cultura sob diferentes cenários climáticos futuros. Não é permitida nenhuma alteração nos métodos de cultivo ou quaisquer outros fatores que possam influenciar a produtividade. Assim, todas as alterações podem ser atribuídas às variáveis de interesse, ou seja, temperatura, precipitação ou níveis de $\mathrm{CO}_{2}$ na atmosfera, possibilitando estimativas não viesadas. As mudanças na produtividade são então utilizadas em modelos econômicos que estimam produção e preços (MENDELSOHN, 2000). Essa modelagem teve ampla aplicação para a agricultura americana, sendo que alguns estudos seminais foram os de Adams et al. (1989, 1990, 1995 e 1998). No Brasil, podem ser citados os estudos de Siqueira et al. (1994) e Nobre et al. (2005) e Embrapa (2008).

Siqueira et al. (1994) simularam o impacto do efeito estufa sobre a produção brasileira de trigo, milho e soja. Os resultados indicaram redução no ciclo e na produção de trigo e milho, sendo a soja menos afetada devido ao efeito benéfico da maior concentração de $\mathrm{CO}_{2}$ (fertilização por carbono). As regiões Nordeste e Centro-Oeste foram identificadas como as mais vulneráveis.

As estimativas de Nobre et al. (2005) indicam que, com aumento de $1^{\circ} \mathrm{C}$ na temperatura, haveria redução das áreas de cultivo do café nos estados de Minas Gerais, Paraná e São Paulo, cujo impacto poderia chegar à diminuição de quatro milhões de sacas/ano. Diferentemente de Siqueira et al. (1994), os autores estimaram que o maior impacto relativo devido ao aumento de temperatura seria para a soja, com redução de até $60 \%$ na área de plantio potencial. 
As projeções do estudo da Embrapa (2008) são bastante pessimistas e indicam que o aquecimento global pode provocar perdas nas safras de grãos de R\$ 7,4 bilhões em 2020 e de até R\$ 14 bilhões em 2070. De acordo com o estudo, a soja deverá ser a cultura mais afetada, com perdas que podem chegar a $40 \%$; o café arábica poderá perder até $33 \%$ da área de baixo risco em São Paulo e Minas Gerais, embora possa haver um aumento de produção no Sul do país; milho, arroz, feijão, algodão e girassol sofrerão perdas significativas da produção, principalmente no Nordeste; por fim, a produção de cana-de-açúcar será beneficiada, podendo até mesmo dobrar.

Em geral, as estimativas baseadas em funções de produção não levam em conta as possibilidades de adaptação aos efeitos da mudança climática. A principal consequência de não considerar as estratégias adaptativas é a superestimação dos impactos das mudanças do clima sobre o setor agrícola. Contudo, alguns esforços para corrigir essa limitação foram feitos e estão relacionados com a simulação de métodos alternativos de cultivo, mudança nas culturas, nas épocas de plantio e colheita, entre outros, como nos estudos de Kaiser et al. (1993) e Easterling et al. (1993), ou com a inclusão de livre mobilidade do insumo trabalho ao estimar a função de produção, como em Assunção e Chein (2008). Infelizmente, essas alternativas não consideram importantes fatores relacionados às decisões de produtores, como, por exemplo, limitações de capital.

Procurando corrigir a sobre-estimação característica dos resultados baseados na abordagem da função de produção, Mendelsohn et al. (1994) desenvolveram a metodologia que ficou conhecida como modelo Ricardiano $^{5}$ (ou hedônico). De acordo com os autores, o objetivo é estimar como o clima, em diferentes localidades, afeta os ganhos dos produtores. O modelo incorpora a adaptação indiretamente, considerando que os produtores utilizarão suas terras sempre do modo mais lucrativo, levando em conta as condições econômicas e climáticas. Assume-se que, para maximizar seus lucros, os agricultores tomam as variáveis climáticas como dadas e ajustam seus insumos e a forma de exploração

5 A metodologia recebeu esse nome porque é baseada nas observações de David Ricardo, segundo o qual o valor da terra seria o reflexo de sua produtividade (sob competição perfeita). 
da terra. Portanto, para cada cenário climático, haverá um tipo específico de organização da produção; na medida em que o clima se modifica, o produtor também altera suas atividades. A representação do modelo pode ser vista na Figura 1.

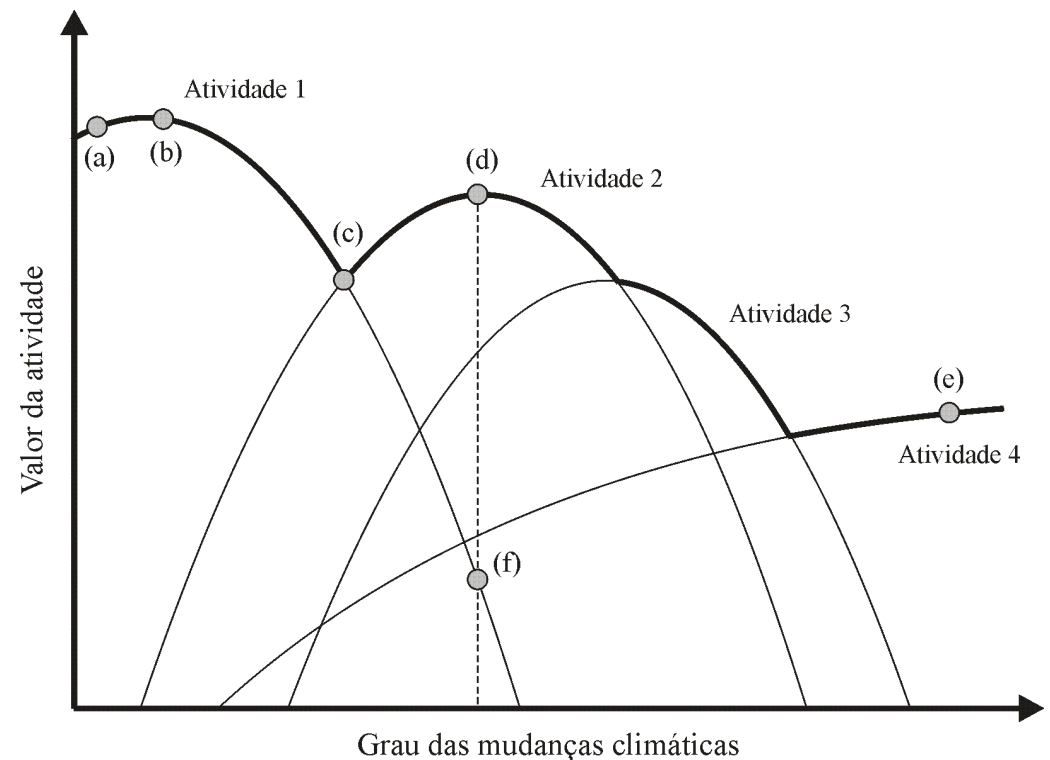

Figura 1 - Representação do modelo hedônico.

Fonte: Adaptado de Mendelsohn et al. (1994).

A Figura 1 apresenta as funções de produção estimadas para quatro atividades agrícolas diferentes. Assume-se que cada uma dessas funções represente adequadamente o comportamento do valor econômico da atividade, considerando o grau das mudanças climáticas. No caso da Atividade 1, seu valor aumenta a partir de baixa intensidade da mudança climática até atingir o valor máximo no ponto (b), decrescendo a partir de então. Segundo Mendelsohn et al. (1994), o produtor perceberá a redução de seus ganhos e, hipoteticamente, a partir do ponto (c), passará a praticar outra atividade, possibilitando que seus lucros voltem a crescer. Note que no ponto (d) o agricultor obtém lucro máximo com a Atividade 2. No entanto, a abordagem da função de produção não consideraria essa 
mudança e, consequentemente, estimaria que os ganhos seriam dados pelo ponto (f). Esse raciocínio permite compreender como o modelo de Mendelsohn et al. (1994) corrige o viés causado pela análise baseada em funções de produção. Em outras palavras, o modelo hedônico parte da pressuposição de que sempre que as alterações no clima tornarem os ganhos decrescentes, o produtor racional procurará se adaptar, alterando suas técnicas ou praticando outras formas de exploração agrícola.

Para considerar a dinâmica descrita pela Figura 1, no modelo de Mendelsohn et al. (1994) analisa-se como a mudança climática afeta o valor da terra dos produtores, ao invés de estimar a produtividade de culturas específicas. Para isso, pressupõe-se que, sob mercados competitivos, o valor da terra será igual à receita líquida do seu melhor uso, já que os produtores são racionais e sempre praticarão a atividade mais lucrativa. Ao considerar o preço da terra, o modelo estima diretamente os impactos do clima sobre a produtividade de diferentes culturas e, indiretamente, a substituição de insumos e a introdução de diferentes técnicas produtivas e outras potenciais adaptações. Se os mercados de terra funcionarem adequadamente, o modelo Ricardiano permitirá medir o impacto das mudanças climáticas sobre o valor econômico de diferentes atividades.

Empiricamente, no modelo Ricardiano, estima-se uma regressão do valor da terra em função de variáveis climáticas, socioeconômicas e agronômicas:

$$
y_{i t}=X_{i t}^{\prime} \beta+\sum_{j} \theta_{j} f_{j}\left(\bar{W}_{i j}\right)+\varepsilon_{i t}
$$

em que $y_{i t}$ refere-se ao valor da terra em cada localidade $i$ no período $t$; $X_{i t}$ é um vetor de determinantes observáveis do valor da terra, como, por exemplo, o tipo e a qualidade do solo (o subscrito $t$ indica que alguns podem variar ao longo do tempo); $\bar{W}_{i j}$ representa as diversas 
variáveis climáticas $j$ para cada localidade $i$ (no modelo original, médias de temperatura e precipitação); $f$ indica a forma funcional para as varáveis climáticas (a maior parte da literatura utiliza termos lineares e quadráticos); $\varepsilon_{i t}$ é o termo de erro estocástico, formado por um componente permanente e específico de cada localidade $\alpha_{i}$ e um componente transitório que varia ao longo do tempo $u_{i t}$ (ou seja, $\left.\varepsilon_{i t}=\alpha_{i}+u_{i}\right)$; e $\beta$ e $\theta$ são os coeficientes que serão estimados. As estimativas de $\theta$ são utilizadas para simular o impacto do clima futuro sobre o valor da terra (DESCHÊNES; GREENSTONE, 2007).

Após o estudo pioneiro de Mendelsohn et al. (1994), diversos autores vêm utilizando o modelo Ricardiano, fazendo com que este seja, até então, o método mais empregado na literatura. Segundo Nordhaus e Boyer (2000), essa metodologia fez com que a análise de impactos das mudanças climáticas sobre o setor agrícola se tornasse menos pessimista. Contudo, os resultados para uma mesma região nem sempre são compatíveis. Para os Estados Unidos, por exemplo, Mendelsohn et al. (1994) sugerem impactos significativamente baixos ou até mesmo benefícios para a agricultura, ao passo que Schlenker et al. (2005), Schlenker et al. (2006), Schlenker e Roberts (2008) e Fisher et al. (2009) concluem que o setor terá sérios prejuízos.

Nas análises da agricultura brasileira, alguns exemplos de utilização do modelo hedônico são os estudos de Sanghi et al. (1997), Ávila et al. (2006) e Féres et al. (2008) e Speranza e Féres (2010). Sanghi et al. (1997) analisaram os impactos da mudança climática sobre a produção agrícola nacional, utilizando dados dos censos agropecuários de 1970 , 1975, 1980 e 1985 e baseando-se em previsões de aumento de $2,5^{\circ} \mathrm{C}$ na temperatura e de $7 \%$ na precipitação. $\mathrm{O}$ resultado principal do estudo foi um impacto líquido negativo, ainda que tenham sido identificadas diferenças sazonais e regionais: as temperaturas de dezembro causariam os piores impactos e os maiores prejuízos seriam para o Centro-Oeste, em contraste com os possíveis benefícios para a região Sul. 
O estudo de Ávila et al. (2006) se concentrou no impacto sobre o valor da terra, distinguindo entre pequenos e grandes produtores. Quando consideraram um cenário climático menos pessimista, caracterizado por aumento de $2^{\circ} \mathrm{C}$ na temperatura e maior precipitação, seus resultados indicaram que em 2020 haveria um impacto positivo, mas que provavelmente seria revertido em 2060 e 2100 . No entanto, caso haja $5^{\circ} \mathrm{C}$ de aumento na temperatura e redução na precipitação, em 2100 as perdas poderiam variar entre $9 \%$ e $31 \%$ para pequenos produtores e entre $47 \%$ e $80 \%$ para os grandes.

Os resultados de Féres et al. (2008) indicaram que o valor da terra poderá decrescer cerca de $21 \%$ no período de 2040 a 2069 e até $42 \%$ entre 2070 e 2099. Segundo os autores, a magnitude das perdas estimadas pode ter sido superestimada, pois o modelo hedônico teve como base os anos de 1970 a 1985. Dessa forma, não foi captado o progresso tecnológico pelo qual o setor agrícola nacional passou após meados da década de 1980 .

Speranza e Féres (2010) estimaram o modelo Ricardiano para diferentes tamanhos de propriedade (pequenos, médios e grandes produtores), considerando sua localização ao longo dos biomas brasileiros (caatinga, cerrado, mata atlântica etc.). As estimativas para o país como um todo indicaram perdas que podem variar de $10 \%$ a $14 \%$ para cenários de mudança climática compreendidos entre 2010 e 2099. Os maiores impactos negativos identificados foram mais elevados para os pequenos produtores. Considerando que, entre as diferentes classes de tamanho de propriedade consideradas, esta é a que contém o maior número de estabelecimentos agrícolas, os autores concluíram que o país poderá ter sérios prejuízos.

Apesar da larga utilização do modelo Ricardiano, é preciso destacar suas limitações. Por se tratar de uma análise de equilíbrio parcial, na qual os preços são mantidos fixos, ignora a potencial perda de bem-estar devida à redução no excedente do consumidor que resultaria, por exemplo, de uma queda na oferta. Também não considera que os preços dos insumos se modificarão, o que pode aumentar os efeitos negativos das alterações 
no clima (CLINE, 1996). Além disso, o modelo assume que o produtor irá alterar suas técnicas e/ou culturas produzidas, mas ignora o processo de substituição, desconsiderando a existência de custos nesse processo. Por fim, dado que é um modelo cross-section, não é possível considerar os possíveis efeitos benéficos da fertilização por carbono. Esses aspectos negativos da metodologia tendem a subestimar os efeitos da mudança climática.

Em termos da estimação econométrica, Deschênes e Greenstone (2007) apresentam outra limitação do modelo Ricardiano. Os autores questionam a robustez das estimativas, afirmando que características específicas de cada localidade (ou qualquer outra variável), que não se modificam ao longo do tempo e que afetam os valores da terra, são variáveis omitidas na estimação. Dessa forma, pode haver correlação com as variáveis climáticas, tornando os coeficientes do modelo viesados. Em termos econométricos, os autores estão enfatizando que a estimação consistente de $\theta$ requer que seja satisfeita a condição de ortogonalidade para cada variável climática, ou seja, $E\left[f_{j}\left(\bar{W}_{i j}\right) \varepsilon_{i t} \mid X_{i t}\right]=0$. Entretanto, essa condição não será válida se existirem fatores permanentes $\left(\alpha_{i}\right)$ e/ou transitórios $\left(u_{i t}\right)$, que sejam correlacionados com as variáveis climáticas.

Para eliminar o viés de variável omitida, Deschênes e Greenstone (2007) propõem um modelo de dados em painel com efeitos fixos, que permite controlar as especificidades de cada localidade. Para que essa abordagem seja implementada, não é possível usar variáveis climáticas semelhantes às utilizadas no modelo Ricardiano, dado que elas são fixas ao longo do horizonte temporal do painel e, assim, perfeitamente colineares com os efeitos fixos de cada localidade. Por essa razão, devem ser usados dados anuais de tempo (temperatura e precipitação). A variável dependente usada é o lucro anual (receita com as vendas menos os custos de produção). Para justificar a inclusão de lucros agrícolas, ao invés de valores da terra, pressupõe-se que, como as variações climáticas afetam as receitas e os custos dos produtores, isso será refletido nos lucros. Portanto, é medido o efeito de variações de temperatura e de precipitação 
sobre os lucros de curto prazo, ao invés do efeito de variações climáticas sobre os valores de longo prazo da terra. Os choques climáticos são modelados como desvios em relação à média histórica das variáveis de temperatura e precipitação.

A equação 2 representa a especificação econométrica do modelo de Deschênes e Greenstone (2007):

$$
y_{i t}=\alpha_{i}+\gamma_{t}+X_{i t}^{\prime} \beta+\sum_{j} \theta_{j} f_{j}\left(W_{i j}\right)+u_{i t}
$$

em que $y_{i t}$ se refere aos lucros agrícolas, em cada localidade $i$ no período $t$; $X_{i t}$ é um vetor de determinantes observáveis dos lucros agrícolas, alguns dos quais variam no tempo; $\alpha_{i}$ capta os fatores específicos de cada localidade, que não variam com o tempo e que afetam $y_{i t} ; \gamma_{t}$ controla as diferenças anuais em $y_{i t}$ que são comuns a todas as localidades (supõe-se que essas dummies temporais incorporem determinantes da lucratividade agrícola, como progresso tecnológico, por exemplo); $W_{i j}$ representa realizações anuais das variáveis climáticas (temperatura e precipitação); e $u_{i t}$ é o termo de erro estocástico.

A validade das inferências a respeito do impacto das mudanças climáticas no setor agrícola, baseadas nesse modelo, depende de que sejam obtidas estimativas não viesadas do vetor $\theta$. Assim como no modelo Ricardiano, isso requer que seja válida a condição de ortogonalidade, que, nesse caso, será $E\left[f_{i}\left(W_{i j}\right) u_{i t} \mid X_{i t}, \alpha_{i}, \gamma_{t}\right]=0$. Para garantir a condição de ortogonalidade, todas as variáveis climáticas $W_{i j}$ são introduzidas como desvios em relação às suas médias (desvios da temperatura e precipitação observados em determinado ano em relação às suas médias históricas). Dessa forma, supõe-se que as variáveis de clima não serão correlacionadas 
com os determinantes não observados da lucratividade agrícola que fazem parte de $u_{i t}$, o que é uma possível solução ao problema de viés de variável omitida apresentado pela abordagem hedônica ${ }^{6}$.

Os resultados de Deschênes e Greenstone (2007) indicam que, nos EUA, as mudanças no clima tendem a aumentar os lucros agrícolas anuais em cerca de US\$ 1,3 bilhão (em dólares de 2002) ou em 4\% no fim do século XXI. Segundo os autores, embora o efeito seja pequeno, há considerável heterogeneidade entre os estados americanos, sendo que alguns têm ganhos e outros, perdas expressivas. Esses resultados, no entanto, foram questionados por Fisher et al. (2009), que identificaram erros nas estimativas e, após corrigi-las, concluíram que a agricultura norte-americana sofrerá fortes impactos negativos ${ }^{7}$.

Féres et al. (2008) utilizaram o modelo de Deschênes e Greenstone (2007) para avaliar os efeitos da mudança climática sobre a produção agrícola brasileira. Os autores consideraram como variável dependente os lucros do setor, reportados nos censos agropecuários de 1970, 1975, 1980, 1985 e 1995/96. Os resultados do estudo indicaram que, a médio prazo (período de 2040 a 2069), mesmo considerando um cenário pessimista, haveria perda líquida de apenas 3,7\% para a agricultura do país. No entanto, quando se considera o longo prazo (2070 a 2099), as perdas seriam substanciais e poderiam chegar a $26 \%$. Suas simulações sugerem ainda que os efeitos seriam diferenciados quando se consideram as regiões do Brasil; o Norte e o Centro-Oeste sofreriam as maiores perdas, mas o Sudeste e o Sul teriam ganhos em termos de aumento da lucratividade.

Mesmo oferecendo vantagens econométricas em relação à abordagem hedônica, a metodologia de Deschênes e Greenstone (2007) apresenta algumas limitações. De modo similar às funções de produção discutidas

6 Segundo Féres et al. (2009), os desvios não podem ser antecipados pelos produtores, e, dessa forma, são supostamente ortogonais aos determinantes não observáveis dos lucros agrícolas.

7 Os erros se referem à especificação e cálculo das variáveis climáticas. Para fazer as simulações de impacto, Fischer et al. (2009) utilizaram o modelo climático Hadley III, que é menos otimista que o Hadley II (usado por Deschênes e Greenstone, 2007). 
acima, o modelo não permite a inclusão de estratégias adaptativas em resposta às variações no clima. O conceito de lucro também é problemático. Conforme Fisher et al. (2009), ao considerar como lucro o valor das receitas líquidas, deixa-se de levar em conta a possível existência de estoques. Em anos de alta produtividade, os produtores podem acumular estoques que, posteriormente, serão desfeitos quando as condições de produção forem ruins. Os estoques são uma forma de reduzir prejuízos relacionados, por exemplo, a variações climáticas e, portanto, criam uma desconexão entre a medida de lucro utilizada e o impacto da mudança no clima.

Todavia, a principal limitação do modelo de efeitos fixos está relacionada à modelagem do choque climático como um desvio em relação à média históricas das variáveis de temperatura e precipitação. Segundo Fisher et al. (2009), esse é um choque temporário, relativo às condições do tempo para um período particular, ao passo que mudança climática é um fenômeno de longo prazo. Dessa forma, os parâmetros estimados podem não refletir as respostas do setor agrícola aos efeitos permanentes das alterações futuras do clima global.

Após a apresentação da abordagem da função de produção, do modelo Ricardiano de Mendelsohn et al. (1994) e do método de efeitos fixos de Deschênes e Greenstone (2007), é possível perceber a evolução das metodologias que visam a estimar o efeito das mudanças climáticas sobre o setor agrícola. Tais modelos abriram caminho para o desenvolvimento de estudos que consideram as ações que os produtores podem empreender para se adaptar, modelando a adaptação explicitamente como variável de controle.

\section{Modelos com adaptação}

Segundo Seo e Mendelsohn (2008a), para quantificar adequadamente os impactos das alterações climáticas sobre a agricultura, é preciso levar em conta as estratégias de adaptação. As análises não podem 
simplesmente estimar como uma cultura específica será afetada, mas devem reconhecer que os produtores irão modificar suas decisões de produção para maximizar o lucro conforme cada cenário climático. Estudos que assumem que os produtores continuarão cultivando a(s) mesma(s) cultura(s), sem alteração de suas técnicas produtivas, certamente superestimam os prejuízos.

A capacidade de adaptar-se é dinâmica e é influenciada pela base produtiva da sociedade, em particular, pelos bens de capital, capital humano, instituições, tecnologia e disponibilidade de recursos naturais (IPCC, 2007). As principais estratégias adaptativas para o setor agrícola incluem diversificação de culturas, alterações das datas de plantio e colheita, aumento do uso de irrigação, implementação de técnicas de conservação de solo, sombreamento e abrigo e, por fim, melhoramento genético, por meio do desenvolvimento de cultivares mais resistentes à seca e, ou, ao estresse hídrico. Deve-se considerar que o planejamento de ações de adaptação climática requer, necessariamente, a análise das opções de decisão, levando em conta as possibilidades e limitações de cada técnica assim como as incertezas associadas às mudanças climáticas (PIDGEON; FISCHHOFF, 2011).

Uma das formas de considerar adaptação é por meio da análise da influência da mudança climática nos padrões de uso da terra. Tratase de analisar, explicitamente, como o clima influencia o processo de mudança de uma atividade para outra, descrito na Figura 1. A análise foi desenvolvida inicialmente por Evenson e Alves (1998) e, posteriormente, aprimorada por Anderson e Reis (2007) e Féres et al. (2009). O modelo é derivado do problema de maximização de lucro do produtor rural, que decide a alocação de suas terras entre alternativos tipos de uso, como pastagens, agricultura temporária ou permanente, florestas etc. Segundo Féres et al. (2009), a partir dos preços dos produtos, do custo dos insumos e das características agronômicas e climáticas, o produtor escolherá a quantidade de área a ser destinada a cada uso, procurando maximizar seu lucro total, respeitando a restrição de que a soma das áreas alocadas para cada atividade não ultrapasse a área total de sua propriedade: 
$\underset{n_{1}, n_{2}, \ldots, n_{m}}{\operatorname{Max}}\left(\sum_{i=1}^{m} \Pi_{i}\left(P_{q i}, P_{x i}, n_{i}, X\right) \cdot \sum_{i=1}^{m} n_{i}=N\right)$

em que o índice $i$ é o tipo de atividade de atividade desenvolvida; $m$ é o número total de usos da terra; $n_{i}$ refere-se à área alocada para o uso $i$; $\Pi_{i}$ representa o lucro obtido com a atividade $i ; P_{q i}$ é o preço do produto relativo à atividade $i ; P_{x i}$ é o vetor de preços dos insumos; $X$ é o vetor de variáveis que influenciam a lucratividade; e $N$ é a área total do estabelecimento agrícola.

Evenson e Alves (1998), Anderson e Reis (2007) e de Féres et al. (2009) analisaram as mudanças no padrão de uso da terra nos estabelecimentos agrícolas brasileiros, considerando lavouras, pastagens e floresta. A conclusão comum aos três estudos foi que as mudanças climáticas potencialmente levarão ao aumento da utilização do solo para agricultura e pastagens em detrimento da área de floresta (natural ou plantada). Uma importante implicação disso é que o processo de adaptação às alterações no clima no Brasil se dará com base em aumento do desmatamento, um dos principais responsáveis pelas emissões de $\mathrm{CO}_{2}$, contribuindo, portanto, para o agravamento do aquecimento global.

Outra metodologia que procura incluir medidas adaptativas é o modelo Ricardiano estrutural. De acordo com Seo e Mendelsohn (2008b), diferentemente da abordagem tradicional de Mendelsohn et al. (1994), que faz referência à adaptação apenas indiretamente, o modelo estrutural a considera explicitamente e quantifica sua influência sobre os impactos das mudanças climáticas no setor agrícola. Em outras palavras, no modelo estrutural, o agente faz uma escolha entre múltiplas alternativas de adaptação no primeiro estágio e maximiza seu lucro no segundo estágio, condicional à seleção inicial. Estima-se, dessa forma, não apenas o efeito do clima sobre o desempenho dos produtores, mas também sua influência na opção por determinada medida adaptativa. 
Há diversas versões do modelo Ricardiano estrutural, sendo que a decisão da medida adaptativa pode ser caracterizada por um processo binário ou múltiplo. Essa metodologia já foi utilizada para analisar a escolha de cultivos alternativos, pecuária e uso de irrigação e tem sido implementada, em grande parte, no estudo de países em desenvolvimento da África e do continente americano (KURUKULASURIYA; MENDELSOHN, 2007 e 2008; MENDELSOHN; SEO, 2007; SEO; MENDELSOHN, 2008b; SEO, 2010 e 2011). Os resultados de alguns estudos realizados para a América Latina serão apresentados a seguir.

Mendelsohn e Seo (2007) analisaram se os produtores, em face de diferentes cenários climáticos, tendem a escolher distintas formas de exploração agrícola. Foram considerados cinco tipo de organização da produção: agricultura de sequeiro, agricultura irrigada, pecuária, ou combinações de pecuária e agricultura (de sequeiro e irrigada). O estudo conclui que todos os tipos de produtores irão sofrer prejuízos devidos às mudanças esperadas no clima, mas a agricultura de sequeiro será a mais prejudicada.

A análise desenvolvida por Seo (2010) considerou a possibilidade de escolher entre a agricultura, a pecuária ou a combinação de ambas. Seus resultados mostraram que em regiões mais quentes há predominância de sistemas mistos. Num cenário climático mais seco e com maiores temperaturas, o valor da terra dos três sistemas tende a decrescer, mas os produtores que combinam agricultura e pecuária serão menos afetados. A principal conclusão do estudo é que, na ausência de adaptação, os impactos das mudanças climáticas tendem a ser mais severos.

Diferentemente dos estudos anteriores, o foco do trabalho de Seo (2011) foi a utilização de esquemas de irrigação públicos e privados como medida adaptativa. $\mathrm{O}$ autor analisou a influência do clima na escolha de cada tipo de esquema no primeiro estágio e, posteriormente, no valor da terra. De acordo com os resultados apresentados, a irrigação privada

8 Todos os estudos referentes à América Latina descritos nessa seção foram desenvolvidos utilizando a mesma base de dados. A amostra contém dados da Argentina, Brasil, Chile, Colômbia, Equador, Uruguai e Venezuela. 
ocorre em resposta a aumentos de temperatura, ao passo que os esquemas públicos são desenvolvidos devido à escassez de água. Tanto a produção irrigada quanto a produção de sequeiro irão sofrer prejuízos com as mudanças climáticas, no entanto, esperam-se menos efeitos adversos para a primeira.

De modo geral, pode-se inferir que produtores adaptados, seja pela prática de mais de um tipo de exploração agrícola, pelo uso de irrigação ou de sementes geneticamente melhoradas etc., serão menos prejudicados pelas mudanças climáticas. Essa conclusão empírica confirma que estudos que não levam em conta as estratégias adaptativas tendem a superestimar os impactos adversos das mudanças climáticas. Além disso, sob outra perspectiva, desconsiderar a adaptação corresponde a subestimar a capacidade de percepção e tomada de decisões dos agricultores. Ainda que persistam muitas incertezas a respeito da magnitude do fenômeno, há cada vez mais consenso de que as mudanças climáticas são reais e podem causar severos impactos negativos no setor agrícola. Assim, para tornar as análises mais realistas, é fundamental desenvolver modelos que considerem explicitamente a adaptação.

\section{Considerações finais}

Mesmo havendo diferenças de entendimento sobre a forma de mensurar os impactos, verifica-se que a maior parte dos pesquisadores acredita que as nações em desenvolvimento serão as mais afetadas negativamente. Em primeiro lugar, por ser a agropecuária atividade econômica responsável por expressiva parcela do Produto Interno (PIB) desses países. Além disso, muitos deles apresentam partes significativas de seus territórios em áreas onde as temperaturas já estão próximas ou acima dos níveis ótimos para a agricultura. Por essa razão, até mesmo elevações moderadas na temperatura levarão a perdas de produtividade.

Deve-se ressaltar, entretanto, a necessidade de incluir estratégias de adaptação nos modelos de análise. Tais medidas têm sido cada vez 
mais enfatizadas de modo a formar uma resposta política às mudanças climáticas mais abrangente A adaptação é necessária pois, mesmo que as emissões de gases de efeito estufa se reduzam nas próximas décadas, o estoque desses gases tende a permanecer por muitos anos na atmosfera, dando continuidade às alterações do clima. Portanto, a adaptação é crucial para lidar com os impactos inevitáveis das mudanças no clima.

\section{Referências}

ADAMS, R.; GLYER, D.; McCARL, B. The economic effects of climate change in US agriculture: a preliminary assessment. In: TIRPAK, D.; SMITH, J. (Eds.). The potential effects of global climate change in the United States: report to congress. Washington, DC: United States Environmental Protection Agency, 1989.

ADAMS, R.; ROSENZWEIG, C.; PEART, R. M.; RITCHIE, J. T.; MCCARL, B.; GLYER, D.; CURRY, R. B.; JONES, J. W.; BOOTE; K. J.; ALLEN JR, L. H. Global climate change and US agriculture. Nature, v. 345, n. 6272, p. 219-224, 1990.

ADAMS, R.; FLEMING, R. A.; CHANG, C; McCARL, B.; ROSENZWEIG, C. A reassessment of the economic effects of global climate in US agriculture. Climatic Change, v. 30, n. 2, p. 147-167, 1995.

ADAMS, R.; McCARL, B.; SEGERSON, K.; ROSENZWEIG, C.; BRYANT, K. J.; DIXON, B. L.; CONNER, R.; EVENSON, R. E.; OJIMA, D. The economic effects of climate change on US agriculture. In: MENDELSOHN, R.; NEUMANN, J. (Eds.). The economic impact of climate on the economy of the United States. Cambridge, UK: Cambridge University Press, 1998.

ANDERSON, K; REIS, E. The effects of climate change on Brazilian agricultural profitability and land use: cross-sectional model with census data. Final report to WHRC/IPAM for LBA project Global Warming, Land Use, and Land Cover Changes in Brazil. 2007. 
ASSUNÇÃO, J. J.; CHEIN, F. Climate change, agricultural productivity and poverty. In: Latin American and Caribbean Economic Association Annual Meeting - LACEA, 2008, Rio de Janeiro. Anais... Rio de Janeiro: LACEA, 2008.

ÁVILA, A. F. D; IRIAS, L. J. M.; LIMA, M. Impacto das mudanças climáticas na agricultura brasileira. Brasília: Embrapa, 2006.

CLINE, W. R. The impact of global warming on agriculture: comment. The American Economic Review, v. 86, n. 5, p. 1309-1311, 1996.

DÊSCHENES, O.; GREENSTONE, M. The economic impacts of climate change: evidence from agricultural output and random fluctuations in weather. The American Economic Review, v. 97, n. 1, p. 354-385, 2007.

EASTERLING, W. E.; AGGARWAL, P. K.; BATIMA, P.; BRANDER, L. M.; ERDA, L.; HOWDEN, S. M. Food, fibre and forest products. In: PARRY, M. L.; CANZIANI, O. F.; PALUTIKOF, J. P.; VAN DER LINDEN, P. J.; HANSON, C. E. (Eds.). Climate Change 2007: impacts, adaptation, and vulnerability - contribution of Working Group II to the Fourth Assessment Report of the Intergovernmental Panel on Climate Change. Cambridge, UK: Cambridge University Press, 2007. p. 273-313.

EASTERLING, W. E.; CROSSON, P. R.; ROSENBERG, N. J.; MCKENNEY, M.S.; KATZ, L. A.; LEMON, K. M. Agricultural impacts of and responses to climate change in the Missouri-Iowa-NebraskaKansas (MINK) region. Climatic Change, v. 24, n. 1-2, p. 23-61, 1993.

EMPRESA BRASILEIRA DE PESQUISA AGROPECUÁRIA EMBRAPA. Aquecimento Global e a nova Geografia da Produção agrícola no Brasil. 2008. Disponível em < www.embrapa.br/publicacoes/ tecnico/aquecimentoglobal.pdf $>$. Acesso em: jul. 2009. 
EVENSON, R. E.; ALVES, D. C. O. Technology, climate change, productivity and land use in Brazilian agriculture. Planejamento e Políticas Públicas, v. 18, p. 223-258, 1998.

FÉRES, J.; REIS, E.; SPERANZA, J. Assessing the Impact of Climate Change on the Brazilian Agricultural Sector. In: $16^{\text {th }}$ Annual EAERE Conference, 2008, Gothenburg. Proceedings of the $\mathbf{1 6}^{\text {th }}$ Annual EAERE Conference. Gothenburg: EAERE, 2008.

FÉRES, J.; REIS, E.; SPERANZA, J. Mudanças climáticas globais e seus impactos sobre os padrões de uso do solo no Brasil. In: XLVII Congresso da Sociedade Brasileira de Economia, Administração e Sociologia Rural, 2009, Porto Alegre - RS. Anais... Brasília: SOBER, 2009.

FISHER, A. C.; HANEMANN, W. M.; ROBERTS, M. J.; SCHLENKER, W.; Climate change and agriculture reconsidered. Disponível em: <http://www.columbia.edu/ ws2162/agClimateChange/ agClimateChange.pdf $>$. Acesso em ago. 2009.

GOUVÊA, J. R. F. Mudanças climáticas e a expectativa de seus impactos na cultura da cana-de-açúcar na região de Piracicaba, SP. 98 p. Dissertação (Mestrado em Agronomia) - Escola Superior de Agricultura Luiz de Queiroz, 2008.

HANEMANN, M. What is the economic cost of climate change? 2008. Disponível em: $<\mathrm{http}: / /$ scholarship.org/uc/item/9g11z5cc $>$. Acesso em: dez. 2009.

INTERNATIONAL PANEL ON CLIMATE CHANGE - IPCC. Climate Change 2007: Synthesis Report. Contribution of Working Groups I, II and III to the Fourth Assessment Report of the Intergovernmental Panel on Climate Change. PACHAURI, R. K.; REISINGER, A. (Eds.). Geneva, Switzerland: IPCC, 2007.

KAISER, H. M.; RIHA, S. J.; WILKS, D. S.; ROSSITER, D. G.; SAMPATH, R. K. A farm-level analysis of economics and agronomic 
impacts of gradual warming. American Journal of Agricultural Economics, v. 75, n. 2, p. 387-398, 1993.

KAUFMANN, R. K. The impact of climate change on US agriculture: a response to Mendelsohn et al. Ecological Economics, v. 26, n. 2, p. 113-119, 1994.

KURUKULASURIYA, P.; MENDELSOHN, R. Endogenous irrigation: the impact of climate change on farmers in Africa. World Bank Policy Research Working Paper 4278. 2007. Disponível em: < http://wwwwds.worldbank.org/external/default/WDSContentServer/IW3P/IB/200 7/07/06/000016406_20070706160116/Rendered/PDF/wps4278.pdf>. Acesso em out. 2009.

KURUKULASURIYA, P.; MENDELSOHN, R. Crop switching as a strategy for adapting to climate change. African Journal of Agricultural and Resource Economics, v. 2, n. 1, p. 105-126, 2008.

LONG, S. P.; AINSWORTH, E. A.; ROGERS, A.; ORT, D. R. Rising atmospheric carbon dioxide: plants face the future. Annual Review of Plant Biology, v. 55, n. 1, p. 591-628, 2004.

MENDELSOHN, R. Measuring the effect of climate change on developing country agriculture. In: Food and Agriculture Organization of the United Nations - FAO. Two essays on climate change and agriculture: a developing country perspective. Rome: FAO, 2000, p. 1-32.

MENDELSOHN, R.; NORDHAUS, W.; SHAW, D. The impact of global warming on agriculture: Ricardian analysis. The American Economic Review, v. 84, n. 4, p. 753-771, 1994.

MENDELSOHN, R.; SEO, N. Changing farm types and irrigation as an adaptation to climate change in Latin American agriculture. World Bank Policy Research Working Paper 4161. 2007. Disponível em: $<$ http://www-wds.worldbank.org/servlet/WDSContentServer/WDSP/IB 
/2007/03/06/000016406_20070306145501/Rendered/PDF/wps4161. pdf>. Acesso em out. 2009.

NOBRE, C. A.; ASSAD, E. D.; OYAMA, M. D. Mudança ambiental no Brasil: o impacto do aquecimento global nos ecossistemas da Amazônia e na agricultura. Scientific American Brazil, v. 80, p. 70-75, 2005.

NORDHAUS, W; BOYER, J. Warming the world: economic modeling of global warming. Cambridge: MIT, 2000.

PIDGEON; FISCHHOFF. The role of social and decision sciences in communicating uncertain climate risks. Nature Climate Change, v. 1, n. 1, p. $35-41,2011$.

SANGHI, A.; ALVES, D.; EVENSON, R.; MENDELSOHN, R. Global warming impacts on Brazilian agriculture: estimates of the Ricardian model. Economia Aplicada, v. 1, n. 1, p. 7-33, 1997.

SCHLENKER, W.; HANEMANN, W. M.; FISHER, A. C. Will U.S. agriculture really benefit from global warming? Accounting for irrigation in the hedonic approach. The American Economic Review, v. 95, n. 1, p. 395-406, 2005.

SCHLENKER, W.; HANEMANN, W. M.; FISHER, A. C. The impact of global warming on US agriculture: an econometric analysis of optimal growing conditions. Review of Economics and Statistics, v. 88, n. 1, p. 113-125, 2006.

SCHLENKER, W.; ROBERTS, M. J. Estimating the impact of climate change on crop yields: the importance of nonlinear temperature effects. NBER Working Paper 13799, 2008. Disponível em: <http:// www.nber.org/papers/w13799>. Acesso em out. 2009.

SEO, N. A microeconometric analysis of adapting portfolios to climate change: adoption of agricultural systems in Latin America. Applied Economic Perspectives and Policy, v. 32, n. 3, p. 489-514, 2010. 
SEO, N. An analysis of public adaptation to climate change using agricultural water schemes in South America. Ecological Economics, v. 70, n. 4, p. 825-834, 2011.

SEO, N.; MENDELSOHN, R. An analysis of crop choice: adapting to climate change in South American farms. Ecological Economics, v. 67, n. 1, p. 109-116, 2008a.

SEO, N.; MENDELSOHN, R. Measuring impacts and adaptation to climate change: a structural Ricardian model of African livestock management. Agricultural Economics, v. 38, n. 2, p. 151-165, 2008 b.

SIQUEIRA, O. J. F.; FARIAS, J. R. B.; SANS, L. M. A. Efeitos potenciais de mudanças climáticas globais na agricultura brasileira e estudos de adaptação para trigo milho e soja. Revista Brasileira de Agrometeorologia, v. 2, n. 1, p. 115-129, 1994.

SPERANZA, J. ; FERES, J. 2010. Evaluating the long-term effects of global climate change on Brazilian agriculture according to farm size. Working Paper Series No. 2010-WP19, Laceep, 2010. Disponível em: <http://www.laceep.org/images/stories/working_papers/2010wp19_speranza.pdf $>$. Acesso em jan. 2011.

UNITED NATIONS FRAMEWORK CONVENTION ON CLIMATE CHANGE - UNFCCC. Text of the Convention. Disponível em: $<$ http:// unfecc.int/resource/docs/convkp/conveng.pdf>. Acesso em: out. 2009. 